04

\title{
Флуктуационно-диссипативная теорема и частотные моменты функций реакции плотной плазмы на электромагнитное поле
}

\author{
() Г.А. Павлов \\ Институт проблем химической фризики РАН, \\ 142432 Черноголовка, Московская обл., Россия \\ e-mail: pav14411@yandex.ru
}

Поступило в Редакцию 19 мая 2021 г.

В окончательной редакции 10 ноября 2021 г.

Принято к публикации 11 ноября 2021 г.

Рассмотрены флуктуационно-диссипативная теорема и частотные моменты для квадратичных функций реакции плотной плазмы в постоянном магнитном поле на электромагнитное поле. Изучены частотные моменты соответствующих корреляционных функций. Предложен модельный подход к вычислению квадратичных функций реакции, определяющих нелинейные явления, вызванные квадратичным взаимодействием электромагнитных волн в плотной заряженной среде (кулоновские системы, плазма) в постоянном магнитном поле.

Ключевые слова: плотная плазма, нелинейная флуктуационно-диссипативная теорема, квадратичные функции реакции, нелинейные явления.

DOI: $10.21883 /$ JTF.2022.02.52011.149-21

\section{Введение}

В последнее время интенсивно исследуются нелинейные явления в различных средах (кристаллах, графене, различных смесях, композитах, слабоионизованных газах, суспензиях и т.д.), связанные с нелинейным взаимодействием электромагнитных (ЭМ) волн (см., например, [1-4]). В то же время недостаточно изучены нелинейные явления в плотных заряженных системах (кулоновских системах и плазме), вызванные квадратичной реакцией на электромагнитное поле - генерация второй гармоники (ГВГ), параметрическая генерация излучения (ПГИ) (см. [5] и ссылки там), или термическими возмущениями - множественность тепловых режимов в средах с объемным тепловыделением [6,7]. В плотной плазме ГВГ и ПГИ в лабораторных условиях могут реализоваться в присутствии стационарных относительно сильных магнитных полей [5]. Как показывают исследования (см., например, [5,7-11]), последовательное аналитическое вычисление точных формальных выражений (полученных по теории отклика $[12,13])$ для квадратичных функций реакции (КФР) плотных заряженных сред с сильным межчастичным взаимодействием на возмущения невозможно, компьютерное моделирование точных выражений для КФР даже для модельных кулоновских систем затруднено, поэтому для определения данных функций целесообразно использовать модельные подходы. Одним из вариантов модели может быть применение для функции реакции явной аппроксимации с подгоночными параметрами, которые находятся из точных частотных моментов КФР и соответствующих корреляторов [5-7]. В настоящей работе рассмотрим квадратичную флуктуационно-диссипативную теорему и частотные моменты квадратичных функций реакции на электромагнитное поле плотных заряженных сред в постоянном магнитном поле, являющиеся основой предлагаемой модели.

\section{1. Квадратичная флуктуационно-диссипативная теорема}

Определим точные выражения для квадратичных функции реакции заряженной среды (плотной плазмы) в постоянном магнитном поле с вектор-потенциалом $\mathbf{A}(\mathbf{r})$ на электромагнитное поле по теории нелинейного отклика. При этом используем полный гамильтонианы системы $(\widehat{H})$, среды $\left(H_{0}\right)$ и гамильтониан возмущения $H^{\text {ext }}$, связанный с воздействием на среду внешнего поля $\mathbf{D}(\mathbf{r}, t)$, в известном виде (без учета спина частиц; см., например, [12]).

$$
\begin{gathered}
\widehat{H}=H_{0}+H^{\mathrm{ext}} \\
H_{0}=\Sigma_{\mu} \Sigma_{i} \frac{1}{2 m_{i}}\left[p_{\mu i}-\frac{e_{i}}{c} A_{\mu}\left(\mathbf{r}_{i}\right)\right]^{2}+U\left\{\mathbf{r}_{i}\right\} \\
H^{\mathrm{ext}}=-\Sigma_{i} e_{i} \mathbf{r}_{i} \cdot \mathbf{D}(\mathbf{r}, t) e^{\eta t}
\end{gathered}
$$

Здесь $c, m_{i}, e_{i}, p_{\mu i}, A_{\mu}, U\left\{\mathbf{r}_{i}\right\}$ - соответственно скорость света, масса, заряд, $\mu$-компонента импульса $i$-ой частицы, $\mu-$ компонента вектор-потенциала постоянного внешнего магнитного поля и энергия взаимодействия частиц среды (плазмы) между собой, $\Sigma_{i} e_{i} \mathbf{r}_{i}$ дипольный момент среды, $\eta$ - малая положительная величина, обеспечивающая адиабатичность включения возмущения (и причинность - см. [13]). Запишем для 
общности $H^{\mathrm{ext}}$ в виде

$$
H^{\mathrm{ext}}=-\sum_{j} \int d \mathbf{r} B_{j}(\mathbf{r}) b_{j}^{\mathrm{ext}}(\mathbf{r}, t) .
$$

Здесь $B(\mathbf{r})$ - некоторое наблюдаемое свойство системы (например, плотность объемного заряда, плотность электрического тока, дипольный момент), $b^{\text {ext }}(\mathbf{r}, t)-$ соответствующая обобщенная сила (например, внешнее электрическое поле). Выпишем выражение для отклика некоторого наблюдаемого свойства среды $B(\mathbf{r})$ на возмущение (1) [13]

$$
\begin{aligned}
\langle B\rangle= & \langle B\rangle_{0}+\sum_{n=1}^{\infty} \frac{1}{(i \hbar)^{n}} \int_{-\infty}^{t} \int_{-\infty}^{t_{1}} \ldots \int_{-\infty}^{t_{n-1}} \operatorname{Sp}\left\{B ( \mathbf { r } ) \left[H^{\mathrm{ext}}\left(t_{1}\right)\right.\right. \\
& \left.\times\left[H^{\mathrm{ext}}\left(t_{2}\right) \ldots\left[H^{\mathrm{ext}}\left(t_{n}\right), \rho_{e}\right] \ldots\right]\right\} d t_{1} \ldots d t_{n}
\end{aligned}
$$

Здесь $\langle\ldots\rangle,\langle\ldots\rangle_{0}$ означает усреднение по $\rho$ и $\rho_{e}$ (равновесная матрица плотности) соответственно, [.....] коммутатор. Квадратичный отклик имеет вид

$$
\begin{gathered}
\langle B\rangle^{(2)}=\frac{1}{(i \hbar)^{2}} \int_{-\infty}^{t} \int_{-\infty}^{t_{1}} S p\left\{B(\mathbf{r})\left[H^{\mathrm{ext}}\left(t_{1}\right)\left[H^{\mathrm{ext}}\left(t_{2}, \rho_{e}\right)\right]\right]\right\} d t_{1} d t_{2} \\
\left\langle B_{i}\right\rangle^{(2)}=\sum_{j, k} \int_{-\infty}^{t} \int_{-\infty}^{t_{1}} \hat{\chi}_{i j k}^{(2)}\left(t-t_{1}, t-t_{2} ; \mathbf{r}-\mathbf{r}_{1}, \mathbf{r}-\mathbf{r}_{2}\right) \\
\quad \times b_{j}^{\mathrm{ext}}\left(\mathbf{r}_{1}, t_{1}\right) b_{k}^{\mathrm{ext}}\left(\mathbf{r}_{2}, t_{2}\right) d t_{1} d t_{2} d \mathbf{r}_{1} d \mathbf{r}_{2}
\end{gathered}
$$

в этих выражениях $B(\mathbf{r}, t), H^{\operatorname{ext}}(t)$ - операторы в представлении Гейзенберга $\left(H^{\mathrm{ext}}(t)=e^{i H_{0} t} H^{\mathrm{ext}} e^{-i H_{0} t}\right.$, здесь $H_{0}$ - гамильтониан невозмущенной системы, $\left\{b_{j}, b_{k}-\right.$ возмущения. Выпишем, введя $\theta$-функции, в симметризованном виде (по двум последним индексам) функцию реакции второго порядка (см., например, [7]; $V$ — объем системы):

$$
\begin{aligned}
& \hat{\chi}_{i j k}^{(2)}\left(\mathbf{r}-\mathbf{r}_{1}, t-t_{1}, \mathbf{r}-\mathbf{r}_{2}, t-t_{2}\right)=-\frac{\theta\left(t-t_{1}\right) \theta\left(t-t_{2}\right)}{2 \hbar^{2} V} \\
& \quad \times\left\{\theta\left(t_{1}-t_{2}\right)\left\langle\left[\left[B_{i}(\mathbf{r}, t), B_{j}\left(\mathbf{r}_{1}, t\right){ }_{1}\right], B_{k}\left(\mathbf{r}_{2}, t_{2}\right)\right]\right\rangle_{0}\right. \\
& \left.\quad+\theta\left(t_{2}-t_{1}\right)\left\langle\left[\left[B_{i}(\mathbf{r}, t), B_{k}\left(\mathbf{r}_{2}, t_{2}\right)\right], B_{j}\left(\mathbf{r}_{1}, t_{1}\right)\right]\right\rangle_{0}\right\} .
\end{aligned}
$$

Такое определение КФР позволяет в (4) распространить пределы интегрирования по времени до бесконечности. Подставим (5) в (4) и перейдем к фурьепредставлению

$$
\begin{aligned}
\left\langle B_{i}(\mathbf{k}, \omega)\right\rangle^{(2)}= & \frac{1}{V(2 \pi)^{2}} \sum_{k_{1}, k_{2}} \int d \omega_{1} d \omega_{2} \hat{\chi}_{i j k}^{(2)}\left(\mathbf{k}_{1}, \omega_{1} ; \mathbf{k}_{2}, \omega_{2}\right) \\
& \times b_{j}^{\mathrm{ext}}\left(\mathbf{k}_{1}, \omega_{1}\right) b_{k}^{\mathrm{ext}}\left(\mathbf{k}_{2}, \omega_{2}\right) .
\end{aligned}
$$

Выражения для $\hat{\chi}_{i j k}^{(2)}\left(\mathbf{k}_{1}, \omega_{1} ; \mathbf{k}_{2}, \omega_{2}\right)$ и $\left\langle B_{i}(-\mathbf{k},-\omega) \times\right.$ $\left.\times B_{j}\left(\mathbf{k}_{1}, \omega_{1}\right) B_{k}\left(\mathbf{k}_{2}, \omega_{2}\right)\right\rangle_{0}$ имеют вид

$$
\begin{aligned}
& \hat{\chi}_{i j k}^{(2)}\left(\mathbf{k}_{1}, \omega_{1} ; \mathbf{k}_{2}, \omega_{2}\right)=-\frac{N}{2 \hbar^{2} V}\left\{\int \frac{d \omega_{1}^{\prime}}{2 \pi} \int \frac{d \omega_{2}^{\prime}}{2 \pi}\right. \\
& \times\left[1 / i\left(\omega_{1}^{\prime}-\omega_{1}\right)\right]\left[1 / i\left(\omega_{1}^{\prime}+\omega_{2}^{\prime}-\omega_{1}-\omega_{2}\right)\right] \\
& \times[S(012)+S(210)-S(102)-S(201)] \\
& +\int \frac{d \omega_{1}^{\prime}}{2 \pi} \int \frac{d \omega_{2}^{\prime}}{2 \pi}\left[1 / i\left(\omega_{2}^{\prime}-\omega_{2}\right)\right]\left[1 / i\left(\omega_{1}^{\prime}+\omega_{2}^{\prime}-\omega_{1}-\omega_{2}\right)\right] \\
& \times[S(021)+S(120)-S(201)-S(102)]\}, \\
& \left\langle B_{i}(-\mathbf{k},-\omega) B_{j}\left(\mathbf{k}_{1}, \omega_{1}\right) B_{k}\left(\mathbf{k}_{2}, \omega_{2}\right)\right\rangle_{0} \\
& =2 \pi N \delta\left(\omega-\omega_{1}-\omega_{2}\right) \delta\left(\mathbf{k}-\mathbf{k}_{1}-\mathbf{k}_{2}\right) S(012) .
\end{aligned}
$$

В корреляторе $S(a b c)$ набор $\{a, b, c\}$ означает комбинацию $k, \omega$ с соответствующими индексами. Симметрия функции реакции $\hat{\chi}_{i j k}^{(2)}$ относительно двух последних индексов очевидна из определения (5). Для фурье-образа функции реакции $\hat{\chi}_{i j k}^{(2)}\left(\mathbf{k}_{1}, \omega_{1} ; \mathbf{k}_{2}, \omega_{2}\right)$ имеем соотношения

$$
\begin{gathered}
\hat{\chi}_{i k j}^{(2)}\left(\mathbf{k}_{1}, \omega_{1} ; \mathbf{k}_{2}, \omega_{2}\right)=\hat{\chi}_{i j k}^{(2)}\left(\mathbf{k}_{2}, \omega_{2} ; \mathbf{k}_{1}, \omega_{1}\right), \\
\hat{\chi}_{i j k}^{(2)}\left(\mathbf{k}_{1}, \omega_{1} ; \mathbf{k}_{2}, \omega_{2}\right)=\hat{\chi}_{i j k}^{(2)^{*}}\left(-\mathbf{k}_{1},-\omega_{1} ;-\mathbf{k}_{2},-\omega_{2}\right),
\end{gathered}
$$

поскольку $\hat{\chi}_{i j k}^{(2)}\left(\mathbf{r}_{1}, \tau_{1} ; \mathbf{r}_{2}, \tau_{2}\right)$ - действительная функция, что следует из ее феноменологического определения. Действительная и мнимая части $\hat{\chi}_{i j k}^{(2)}\left(\mathbf{k}_{1}, \omega_{1} ; \mathbf{k}_{2}, \omega_{2}\right)$ связаны между собой аналогично соотношениям Крамерса-Кронига для линейных функций реакции [6,7]. Заметим, что можно рассматривать (5), (7) как одни из простейших форм нелинейной флуктуационнодиссипативной теоремы (НФДТ). Применив формулу Сохоцкого $(1 /(x \pm i \delta)=P(1 / x) \mp i \pi \delta(x))$ [13], найдем связи между действительной и мнимой частями функции реакции и корреляторами $S(a b c)$. Составляя комбинации из $\operatorname{Re} \hat{\chi}_{i j k}^{(2)}\left(\mathbf{k}_{1}, \omega_{1} ; \mathbf{k}_{2}, \omega_{2}\right)$ и учитывая свойства циклической перестановки операторов в $S(a b c)$ $\left(\exp \left(-\beta \omega_{a} \hbar\right) S(a b c)=S(b c a)\right)$, получим соотношения между $\operatorname{Re} \hat{\chi}_{i j k}^{(2)}$ и корреляторами $S(a b c)$, которые являются одним из вариантов нелинейной ФДТ (см. [7] и ссылки там):

$$
\begin{aligned}
& -\frac{\operatorname{Re} \hat{\chi}_{i j k}^{(2)}\left(\mathbf{k}_{1}, \omega_{1} ; \mathbf{k}_{2}, \omega_{2}\right)}{\omega_{1} \omega_{2}}-\frac{\operatorname{Re} \hat{\chi}_{i j k}^{(2)}\left(\mathbf{k}_{2}, \omega_{2} ; \mathbf{k}, \omega\right)}{\omega \omega_{2}} \\
& -\frac{\operatorname{Re} \hat{\chi}_{i j k}^{(2)}\left(\mathbf{k}, \omega ; \mathbf{k}_{1}, \omega_{1}\right)}{\omega_{1} \omega}=\frac{-n}{4 \hbar^{2}}\left\{\frac{S(102)+S(201)}{\omega_{1} \omega_{2}}\right. \\
& \left.-\frac{S(012)+S(210)}{\omega \omega_{2}}-\frac{S(021)+S(120)}{\omega_{1} \omega}\right\} .
\end{aligned}
$$

Данные выражения в принципе применимы для вычисления частотных моментов действительной части 
квадратичных функций реакции, поскольку они связывают частотные моменты функций реакции с частотными моментами корреляторов. Вычисление частотных моментов корреляторов рассмотрено в следующем разделе. Задавая КФР, согласно предложенной модели (см. Введение), в виде явной аппроксимации с подгоночными параметрами, можно определить данные параметры сравнением частотных моментов правой и левой частей (10). Подгоночные параметры для КФР зависят от теплофизических характеристик заряженной среды (плотной плазмы, кулоновской системы) и магнитного поля. Заметим, что в линейном случае корреляторы связаны с мнимой частью функции реакции и ФДТ содержит одну функцию реакции и один коррелятор (см., например, [6]). Наиболее просто квадратичная ФДТ выглядит в классическом пределе. Учитывая $\hbar \rightarrow 0$, вместо (10) будем иметь

$$
\begin{aligned}
& -\omega \operatorname{Re} \hat{\chi}_{i j k}^{(2)}\left(\mathbf{k}_{1}, \omega_{1} ; \mathbf{k}_{2}, \omega_{2}\right)-\omega_{1} \operatorname{Re} \hat{\chi}_{i j k}^{(2)}\left(\mathbf{k}_{2}, \omega_{2} ; \mathbf{k}, \omega\right) \\
& -\omega_{2} \operatorname{Re} \hat{\chi}_{i j k}^{(2)}\left(\mathbf{k}, \omega ; \mathbf{k}_{1}, \omega_{1}\right)=\frac{n \beta^{2}}{8}\left[S(210) \omega_{2} \omega_{1}\left(\omega_{1}+\omega_{2}\right)\right. \\
& \left.+S(201) \omega_{2} \omega_{1}\left(\omega_{2}+\omega_{1}\right)\right]=\frac{n \beta^{2}}{4} S(012) \omega \omega_{1} \omega_{2}
\end{aligned}
$$

так как в классическом пределе корреляторы инвариантны относительно перестановки аргументов. Форму (11) используем ниже, так как лабораторная плотная плазма зачастую подчиняется классической статистике [5].

Рассмотрим КФР $\hat{\chi}_{p, i j k}^{(2)}\left(\mathbf{k}_{1}, \omega_{1} ; \mathbf{k}_{2}, \omega_{2}\right)$ и соответствующие корреляторы. Данные КФР определяют квадратичный вклад в поляризацию заряженных сред $\mathbf{P}^{(2)}$ $\left(\mathbf{P}=\Sigma_{i} e_{i} \mathbf{r}_{i} / V\right)$, т.е. ГВГ и ПГИ (см., например, [7] и ссылки там). Из соотношения (см., например, [14])

$$
\dot{\mathbf{P}}=4 \pi \mathbf{j}
$$

можно задать в форме, подобной (7), связанную с $\hat{\chi}_{p, i j k}^{(2)}\left(\mathbf{k}_{1}, \omega_{1} ; \mathbf{k}_{2}, \omega_{2}\right), \quad$ КФР $\hat{\chi}_{J, i j k}^{(2)}\left(\mathbf{k}_{1}, \omega_{1} ; \mathbf{k}_{2}, \omega_{2}\right)$. Тензор $\hat{\chi}_{J, i j k}^{(2)}$ описывает квадратичный вклад $j_{i}^{(2)}(k, \omega)$ в плотность электрического тока заряженной среды под действием ЭМ-поля и, естественно, определяет квадратичную электропроводность $\hat{\sigma}_{i j k}^{(2)}\left(\mathbf{k}_{1}, \omega_{1} ; \mathbf{k}_{2}, \omega_{2}\right)$ (ср. с [7]). В $\hat{\chi}_{J, i j k}^{(2)}\left(\right.$ и $\left.\hat{\sigma}_{i j k}^{(2)}\right), S_{j}(a b c)$ соответствует коррелятору плотностей токов.

В то же время НФДТ в форме (11) неудобна при реализации модельного подхода, заключающегося в применении явной аппроксимации для функций реакции $\hat{\chi}_{p, i j k}^{(2)}$ (например, в форме из [5]) или $\hat{\chi}_{J, i j k}^{(2)}$ с подгоночными параметрами и точных значений частотных моментов соответствующих корреляторов, так как в этом случае тензоры в (11) имеют по 18 компонентов и не инвариантны относительно отображений. Поэтому для $\hat{\chi}_{J, i j k}^{(2)}$ и коррелятора в правой части (11) проведем свертку по волновым векторам $\left(\mathbf{k}, \mathbf{k}_{1}, \mathbf{k}_{2}\right)$ и используем уравнение сохранения для заряда (13) (см., например, [6])

$$
\partial \rho / \partial t=-\operatorname{div} \mathbf{j}
$$

Иными словами, выполнив продольную проекцию тензоров $\hat{\chi}_{J, i j k}^{(2)}$ и коррелятора в (11), получим НФДТ в виде инвариантного соотношения для скаляров: КФР $\hat{\chi}^{(2)}$ плазмы в постоянном магнитном поле на продольное поле в форме $(11)$, описывающей вклад $\rho^{(2)}(k, \omega)$ в суммарную плотность зарядов, и коррелятора плотностей зарядов. Выпишем продольную НФДТ

$$
\begin{aligned}
& -\omega \operatorname{Re} \hat{\chi}^{(2)}(12)-\omega_{1} \operatorname{Re} \hat{\chi}^{(2)}(20)-\omega_{2} \operatorname{Re} \hat{\chi}^{(2)}(01) \\
& =\frac{n \beta^{2}}{4} S_{\rho}(012) \omega \omega_{1} \omega_{2} .
\end{aligned}
$$

В (14) $\hat{\chi}^{(2)}$ - КФР зарядов [7] на внешнее поле, набор $(a, b)$ означает комбинацию $k \omega$ с соответствующими индексами.

Таким образом, используя (14), можно определить „скалярные“ подгоночные параметры для $\hat{\chi}^{(2)}$ заданной согласно предложенной модели в виде явной аппроксимации с подгоночными параметрами - см. Введение), зависящие от теплофизических характеристик заряженной среды (плотной плазмы, кулоновской системы) и магнитного поля. Данные параметры целесообразно использовать для всей линейки КФР $\left(\hat{\chi}_{p, i j k}^{(2)}, \hat{\chi}_{J, i j k}^{(2)}, \hat{\chi}^{(2)}\right)$, взаимосвязанных между собой.

\section{2. Частотные моменты корреляторов заряженной среды в постоянном магнитном поле}

Рассмотрим процедуру вычисления частотных моментов коррелятора плотностей $S_{\rho}(a b c)$ для равновесной плотной плазмы в постоянном магнитном поле, учитывая четность операторов плотности относительно обращения времени. Частотные моменты $S_{\rho}(012)$ в классическом пределе определим согласно соотношению (см., например, [6,7])

$$
\begin{aligned}
& S_{\rho}^{r_{1}, r_{2}}(012)=\int_{-\infty}^{\infty} d \omega_{1} \int_{-\infty}^{\infty} d \omega_{2} \omega_{1}^{r_{1}} \omega_{2}^{r^{2}} S_{c l}(012)=(i)^{r_{1}+r_{2}} \\
& \times\left.\frac{\partial^{r_{1}+r_{2}} S_{c l}(012)}{\partial t_{1}^{r_{1}} \partial t_{2}^{r_{2}}}\right|_{t_{1}-t_{2}=0}=(i)^{r_{1}+r_{2}} \frac{\partial^{r_{1}}}{\partial t_{1}^{r_{1}}} \frac{\partial^{r_{2}}}{\partial t_{2}^{r_{2}}} \\
& \times\left.\frac{1}{N}\left\langle\rho(k, 0) \rho\left(k_{1}, t_{1}\right) \rho\left(k_{2}, t_{2}\right)\right\rangle_{0}\right|_{t_{1}=t_{2}=0} .
\end{aligned}
$$

Плотности в $S_{\rho}^{r_{1}, r_{2}}(012)(\mathbf{k} \| \mathbf{z})$ имеют вид

$$
\begin{gathered}
\rho(\mathbf{k}, 0)=\sum_{t=1}^{N} e z_{i} e^{-i k z_{i}}, \\
\rho\left(\mathbf{k}_{1}, t_{1}\right)=\sum_{j=1}^{N} e z_{j} e^{-i\left(k_{1}^{z} z_{j}\left(t_{1}\right)+k_{1}^{x} x_{j}\left(t_{1}\right)\right)}, \\
\rho\left(\mathbf{k}_{2}, t_{2}\right)=\sum_{k=1}^{N} e z_{k} e^{-i\left(k_{2}^{z} z_{k}\left(t_{2}\right)+k_{2}^{x} x_{k}\left(t_{2}\right)\right)} .
\end{gathered}
$$


В (16) $N$ - полное число частиц в системе. Можно рассмотреть моменты $S_{\rho}^{r_{1}, r_{2}}(012)$ при $r_{1}, r_{2}=0.0 ; 0.2$; $2.2 ; 1.3$ и т. п. согласно (15), (16). Выпишем первые временные производные от плотностей $\left(v_{j}^{z}-z\right.$-координата скорости $j$-ой частицы)

$$
\begin{gathered}
\dot{\rho}_{j}\left(\mathbf{k}_{1}, t_{1}\right)=\sum_{j=1}^{N} e z_{j}\left[-i k_{1}^{z} v_{j}^{z}\left(t_{1}\right)-i k_{1}^{x} v_{j}^{x}\left(t_{1}\right)\right] \\
\times e^{-i\left(k_{1}^{z} z_{j}\left(t_{1}\right)+k_{1}^{x} x_{j}\left(t_{1}\right)\right)}, \\
\dot{\rho}_{k}\left(\mathbf{k}_{2}, t_{2}\right)=\sum_{k=1}^{N} e z_{k}\left[-i k_{2}^{z} v_{k}^{z}\left(t_{2}\right)-i k_{2}^{x} v_{k}^{x}\left(t_{2}\right)\right] \\
\times e^{-i\left(k_{2}^{z} z_{k}\left(t_{2}\right)+k_{2}^{x} x_{k}\left(t_{2}\right)\right)} \\
\ddot{\rho}_{j}\left(\mathbf{k}_{1}, t_{1}\right)=\sum_{j=1}^{N} e z_{j}\left[-i k_{1}^{z} \dot{v}_{j}^{z}\left(t_{1}\right)-i k_{1}^{x} \dot{v}_{j}^{x}\left(t_{1}\right)\right] \\
\times e^{-i\left(k_{1}^{z} z_{j}\left(t_{1}\right)+k_{1}^{x} x_{j}\left(t_{1}\right)\right)}+\sum_{j=1}^{N} e z_{j}\left[-i k_{1}^{x} v_{j}^{x}\left(t_{1}\right)-i k_{1}^{z} v_{j}^{z}\left(t_{1}\right)\right]^{2} \\
\times e^{-i\left(k_{1}^{z} z_{j}\left(t_{1}\right)+k_{1}^{x} x_{j}\left(t_{1}\right)\right)}, \\
\ddot{\rho}_{k}\left(\mathbf{k}_{2}, t_{2}\right)=\sum_{k=1}^{N} e z_{k}\left[-i k_{2}^{z} \dot{v}_{k}^{z}\left(t_{2}\right)-i k_{2}^{x} \dot{v}_{k}^{x}\left(t_{2}\right)\right] \\
\times e^{-i\left(k_{2}^{z} z_{k}\left(t_{2}\right)+k_{2}^{x} x_{k}\left(t_{2}\right)\right)}+\sum_{k=1}^{N} e z_{k}\left[-i k_{2}^{x} v_{k}^{x}\left(t_{2}\right)-i k_{2}^{z} v_{k}^{z}\left(t_{2}\right)\right]^{2} \\
\left.\times k_{2}^{z} z_{k}\left(t_{2}\right)+k_{2}^{x} x_{k}\left(t_{2}\right)\right)
\end{gathered}
$$

Запишем выражение, являющееся частотным интегралом $S_{\rho}^{r_{1}, r_{2}}(012)$ (см. (14)) при $r_{1}, r_{2}=2.2$ :

$$
\begin{aligned}
& \left.(i)^{r_{1}+r_{2}} \frac{\partial^{r_{1}}}{\partial t_{1}^{r_{1}}} \frac{\partial^{r_{2}}}{\partial t_{2}^{r_{2}}} \frac{1}{N}\left\langle\rho(\mathbf{k}, 0) \rho\left(\mathbf{k}_{1}, t_{1}\right) \rho\left(\mathbf{k}_{2}, t_{2}\right)\right\rangle_{0}\right|_{t_{1}-t_{2}=0} \\
& =\left.(i)^{4} \frac{1}{N}\left\langle\rho(\mathbf{k}, 0) \ddot{\rho}\left(\mathbf{k}_{1}, t_{1}\right) \ddot{\rho}\left(\mathbf{k}_{2}, t_{2}\right)\right\rangle_{0}\right|_{t_{1}-t_{2}=0}=\left(i^{4} / N\right) \\
& \times\left\langle\sum _ { i = 1 } ^ { N } e z _ { i } e ^ { - i k z _ { i } } \left\{\sum_{j=1}^{N} e z_{j}\left[-i k_{1}^{z} \dot{v}_{j}^{z}\left(t_{1}\right)-i k_{1}^{x} \dot{v}_{j}^{x}\left(t_{1}\right)\right]\right.\right. \\
& \times e^{-i\left(k_{1}^{z} z_{j}\left(t_{1}\right)+k_{1}^{x} x_{j}\left(t_{1}\right)\right)}+\sum_{j=1}^{N} e z_{j}\left[-i k_{1}^{x} v_{j}^{x}\left(t_{1}\right)-i k_{1}^{z} v_{j}^{z}\left(t_{1}\right)\right]^{2} \\
& \left.\times e^{-i\left(k_{1}^{z} z_{j}\left(t_{1}\right)+k_{1}^{x} x_{j}\left(t_{1}\right)\right)}\right\}\left\{\sum_{k=1}^{N} e z_{k}\left[-i k_{2}^{z} \dot{v}_{k}^{z}\left(t_{2}\right)-i k_{2}^{x} \dot{v}_{k}^{x}\left(t_{2}\right)\right]\right. \\
& \times e^{-i\left(k_{2}^{z} z_{k}\left(t_{2}\right)+k_{2}^{x} x_{k}\left(t_{2}\right)\right)}+\sum_{k=1}^{N} e z_{k}\left[-i k_{2}^{x} v_{k}^{x}\left(t_{2}\right)-i k_{2}^{z} v_{k}^{z}\left(t_{2}\right)\right]^{2} \\
& \left.\left.\times e^{-i\left(k_{2}^{z} z_{k}\left(t_{2}\right)+k_{2}^{x} x_{k}\left(t_{2}\right)\right)}\right\}\right\rangle_{0} \cdot
\end{aligned}
$$

Для проведения расчетов по (15)-(17a) используем следующие определения для усреднения сумм и произведений (см. подробнее [6] и ссылки там, $V$ - объем системы, $F_{s}-s$-частичная корреляционная функция):

$$
\begin{gathered}
\overline{M_{s}}=\frac{N(N-1) \ldots(N-s+1)}{s ! V^{s}} \int_{V} \ldots \int_{V} f\left(r_{1} \ldots r_{s}\right) \\
\times F_{s}\left(r_{1} \ldots r_{s}\right) d r_{1} \ldots d r_{s}, \\
M_{s}=\sum_{1 \leq i_{1} \ldots i_{s} \leq N} f\left(r_{i_{1}} \ldots r_{i_{s}}\right), \\
\left\langle\frac{\partial U}{\partial x_{i}} F\left(r_{1} \ldots r_{N}\right)\right\rangle_{0}=\beta^{-1}\left\langle\partial F\left(r_{1} \ldots r_{N}\right) / \partial x_{i}\right\rangle_{0} .
\end{gathered}
$$

По аналогии с [6] найдем для $S_{\rho}^{2,2}(012)$ (в формулах 1-4 опущены для краткости знаки сумм экспонент и усреднения - cp. с (17a); $H$ - магнитное поле):

$$
\begin{aligned}
& \text { 1. }\left[-i k_{1}^{z} \dot{v}_{j}^{z}-i k_{1}^{x} \dot{v}_{j}^{x}\right]\left[-i k_{2}^{z} \dot{v}_{k}^{z}-i k_{2}^{x} \dot{v}_{k}^{x}\right]=-k_{1}^{z} \dot{v}_{j}^{z} k_{2}^{z} \dot{v}_{k}^{z} \\
& -k_{1}^{x} \dot{v}_{j}^{x} k_{2}^{z} \dot{v}_{k}^{z}-k_{1}^{z} \dot{v}_{j}^{z} k_{2}^{x} \dot{v}_{k}^{x}-k_{1}^{x} \dot{v}_{j}^{x} k_{2}^{x} \dot{v}_{k}^{x}=-k_{1}^{z} k_{2}^{z} \frac{1}{m_{j} m_{k}} \\
& \frac{\partial U}{\partial z_{j}} \frac{\partial U}{\partial z_{k}}+k_{1}^{x} k_{2}^{z}\left(-\frac{1}{m_{j}} \frac{\partial U}{\partial x_{j}}+\frac{e z_{j}}{c m_{j}}\left(v_{j}^{y} H\right)^{x}\right) \frac{1}{m_{k}} \frac{\partial U}{\partial z_{k}} \\
& +k_{1}^{z} k_{2}^{x} \frac{1}{m_{j}} \frac{\partial U}{\partial z_{j}}\left(-\frac{1}{m_{k}} \frac{\partial U}{\partial x_{k}}+\frac{e z_{k}}{c m_{k}}\left(v_{k}^{y} H\right)^{x}\right) \\
& \times-k_{2}^{x}\left(-\frac{1}{m_{j}} \frac{\partial U}{\partial x_{j}}+\frac{e z_{j}}{c m_{j}}\left(v_{j}^{y} H\right)^{x}\right) \\
& \times k_{1}^{x}\left(-\frac{1}{m_{k}} \frac{\partial U}{\partial x_{k}}+\frac{e z_{k}}{c m_{k}}\left(v_{k}^{y} H\right)^{x}\right)
\end{aligned}
$$

2. $\left[-i k_{1}^{z} \dot{v}_{j}^{z}-i k_{1}^{x} \dot{v}_{j}^{x}\right]\left[-i k_{2}^{x} v_{k}^{x}-i k_{2}^{z} v_{k}^{z}\right]^{2}=\left[i k_{1}^{z}\right.$

$$
\begin{aligned}
& \left.\times\left(\frac{1}{m_{j}} \frac{\partial U}{\partial z_{j}}\right)-i k_{1}^{x}\left(-\frac{1}{m_{j}} \frac{\partial U}{\partial x_{j}}+\frac{e z_{j}}{c m_{j}}\left(v_{j}^{y} H\right)^{x}\right)\right] \\
& \times\left[-\left(k_{2}^{x} v_{k}^{x}\right)^{2}-\left(k_{2}^{z} v_{k}^{z}\right)^{2}-2 k_{2}^{x} v_{k}^{x} k_{2}^{z} v_{k}^{z}\right]=-i k_{1}^{z}\left(\frac{1}{m_{j}} \frac{\partial U}{\partial z_{j}}\right) \\
& \times\left[\left(k_{2}^{x} v_{k}^{x}\right)^{2}+\left(k_{2}^{z} v_{k}^{z}\right)^{2}+2 k_{2}^{x} v_{k}^{x} k_{2}^{z} v_{k}^{z}\right]-i k_{1}^{x}\left(-\frac{1}{m_{j}} \frac{\partial U}{\partial x_{j}}\right. \\
& \left.\left.+\frac{e z_{j}}{c m_{j}}\left(v_{j}^{y} H\right)^{x}\right)\right]\left[-\left(k_{2}^{x} v_{k}^{x}\right)^{2}-\left(k_{2}^{z} v_{k}^{z}\right)^{2}-2 k_{2}^{x} v_{k}^{x} k_{2}^{z} v_{k}^{z}\right]
\end{aligned}
$$

3. $\left[-i k_{1}^{x} v_{j}^{x}-i k_{1}^{z} v_{j}^{z}\right]^{2}\left[-i k_{2}^{z} \dot{v}_{k}^{z}-i k_{2}^{x} \dot{v}_{k}^{x}\right]=-\left[\left(k_{1}^{x} v_{j}^{x}\right)^{2}\right.$

$$
+\left(k_{2}^{z} v_{j}^{z}\right)^{2}+2\left(k_{1}^{x} v_{j}^{x}\right)\left(k_{1}^{z} v_{j}^{z}\right]\left[i k_{2}^{z}\left(\frac{1}{m_{k}} \frac{\partial U}{\partial z_{k}}\right)\right.
$$$$
\left.-i k_{2}^{x}\left(-\frac{1}{m_{k}} \frac{\partial U}{\partial x_{k}}+\frac{e z_{k}}{c m_{k}}\left(v_{k}^{y} H\right)^{x}\right)\right] \text {; }
$$

Журнал технической фризики, 2022, том 92, вып. 2 


$$
\begin{aligned}
& \text { 4. }\left[-i k_{1}^{x} v_{j}^{x}-i k_{1}^{z} v_{j}^{z}\right]^{2}\left[-i k_{2}^{x} \dot{v}_{k}^{x}-i k_{2}^{z} \dot{v}_{k}^{z}\right]^{2}=\left[\left(k_{1}^{x} v_{j}^{x}\right)^{2}\right. \\
& +\left(k_{1}^{z} v_{j}^{z}\right)^{2}+2\left(k_{1}^{x} v_{j}^{x}\right)\left(k_{1}^{z} v_{j}^{z}\right]\left[\left(k_{2}^{x} v_{k}^{x}\right)^{2}+\left(k_{2}^{z} v_{k}^{z}\right)^{2}\right. \\
& \left.+2 k_{2}^{x} v_{k}^{x} k_{2}^{z} v_{k}^{z}\right]
\end{aligned}
$$

Проведем усреднение по распределению Гиббса (в классическом пределе гамильтониана $\left.H_{0}\right)$ в (17a), используя выражения $1-4$. После отбора по результатам интегрирования по скоростям член с $H$ останется только в п. 1 , остальные слагаемые в моменте $S_{\rho}^{2,2}(012)$ не зависят от магнитного поля. Выпишем выражения, соответствующие п.п. $1-4$ :

$$
\begin{aligned}
& \text { 1. } \quad-k_{1}^{z} k_{2}^{z} \frac{1}{m_{j} m_{k}} \frac{\partial U}{\partial z_{j}} \frac{\partial U}{\partial z_{k}}+k_{1}^{x} k_{2}^{z}\left(-\frac{1}{m_{j}} \frac{\partial U}{\partial x_{j}}\right. \\
& \left.+\frac{e z_{j}}{c m_{j}}\left(v_{j}^{y} H\right)^{x}\right) \frac{1}{m_{k}} \frac{\partial U}{\partial z_{k}}+k_{1}^{z} k_{2}^{x} \frac{1}{m_{j}} \frac{\partial U}{\partial z_{j}}\left(-\frac{1}{m_{k}} \frac{\partial U}{\partial x_{k}}\right. \\
& \left.+\frac{e z_{k}}{c m_{k}}\left(v_{k}^{y} H\right)^{x}\right)-k_{2}^{x}\left(-\frac{1}{m_{j}} \frac{\partial U}{\partial x_{j}}+\frac{e z_{j}}{c m_{j}}\left(v_{j}^{y} H\right)^{x}\right) \\
& \times k_{1}^{x}\left(-\frac{1}{m_{k}} \frac{\partial U}{\partial x_{k}}+\frac{e z_{k}}{c m_{k}}\left(v_{k}^{y} H\right)^{x}\right) \rightarrow\left(i^{4} / N\right) \\
& \times\left\langle\sum_{i=1}^{N} e z_{i} e^{-i k z_{i}} \sum_{j=1}^{N} e z_{j} e^{-i\left(k_{1}^{z} z_{j}+k_{1}^{x} x_{j}\right)} \sum_{k=1}^{N} e z_{k} e^{-i\left(k_{2}^{z} z_{k}+k_{2}^{x} x_{k}\right)}\right. \\
& \times\left\{-k_{1}^{z} k_{2}^{z} \frac{1}{m_{j} m_{k}} \frac{\partial U}{\partial z_{j}} \frac{\partial U}{\partial z_{k}}+k_{1}^{x} k_{2}^{z}\left(-\frac{1}{m_{j}} \frac{\partial U}{\partial x_{j}}\right) \frac{1}{m_{k}} \frac{\partial U}{\partial z_{k}}\right. \\
& +k_{1}^{z} k_{2}^{x} \frac{1}{m_{j}} \frac{\partial U}{\partial z_{j}}\left(-\frac{1}{m_{k}} \frac{\partial U}{\partial x_{k}}\right)-k_{1}^{x} k_{2}^{x} \frac{1}{m_{j}} \frac{\partial U}{\partial x_{j}} \frac{1}{m_{k}} \frac{\partial U}{\partial x_{k}} \\
& \left.\left.-k_{1}^{x} k_{2}^{x} \frac{e z_{j}}{c m_{j}}\left(v_{j}^{y} H\right)^{x} \frac{e z_{k}}{c m_{k}}\left(v_{k}^{y} H\right)^{x}\right\}\right\rangle_{0} ;
\end{aligned}
$$$$
\text { 2. }\left(i^{4} / N\right)\left\langle\sum_{i=1}^{N} e z_{i} e^{-i k z_{i}} \sum_{j=1}^{N} e z_{j} e^{-i\left(k_{1}^{z} z_{j}+k_{1}^{x} x_{j}\right)}\right.
$$$$
\times \sum_{k=1}^{N} e z_{k} e^{-i\left(k_{2}^{z} z_{k}+k_{2}^{x} x_{k}\right)}\left[-i k_{1}^{z}\left(\frac{1}{m_{j}} \frac{\partial U}{\partial z_{j}}\right)\right.
$$$$
\left.\left.-i k_{1}^{x}\left(\frac{1}{m_{j}} \frac{\partial U}{\partial x_{j}}\right)\right]\left[\left(k_{2}^{x} v_{k}^{x}\right)^{2}+\left(k_{2}^{z} v_{k}^{z}\right)^{2}\right]\right\rangle_{0}
$$

3. $\left(i^{4} / N\right)\left\langle\sum_{i=1}^{N} e z_{i} e^{-i k z_{i}} \sum_{j=1}^{N} e z_{j} e^{-i\left(k_{1}^{z} z_{j}+k_{1}^{x} x_{j}\right)}\right.$

$$
\begin{aligned}
& \times \sum_{k=1}^{N} e z_{k} e^{-i\left(k_{2}^{z} z_{k}+k_{2}^{x} x_{k}\right)}\left[-\left(k_{1}^{x} v_{j}^{x}\right)^{2}-\left(k_{1}^{z} v_{j}^{z}\right)^{2}\right] \\
& \left.\times\left[-i k_{2}^{z}\left(\frac{1}{m_{k}} \frac{\partial U}{\partial z_{k}}\right)-i k_{2}^{x}\left(\frac{1}{m_{k}} \frac{\partial U}{\partial x_{k}}\right)\right]\right\rangle_{0}
\end{aligned}
$$

4. $\left[\left(k_{1}^{x} v_{j}^{x}\right)^{2}+\left(k_{1}^{z} v_{j}^{z}\right)^{2}+2\left(k_{1}^{x} v_{j}^{x}\right)\left(k_{1}^{z} v_{j}^{z}\right]\left[\left(k_{2}^{x} v_{k}^{x}\right)^{2}\right.\right.$

$$
\begin{aligned}
& \left.+\left(k_{2}^{z} v_{k}^{z}\right)^{2}+2 k_{2}^{x} v_{k}^{x} k_{2}^{z} v_{k}^{z}\right] \rightarrow\left(i^{4} N\right)\left\langle\sum_{i=1}^{N} e z_{i} e^{-i k z_{i}}\right. \\
& \times \sum_{j=1}^{N} e z_{j} e^{-i\left(k_{1}^{z} z_{j}+k_{1}^{x} x_{j}\right)} \sum_{k=1}^{N} e z_{k} e^{-i\left(k_{2}^{z} z_{k}+k_{2}^{x} x_{k}\right)}\left\{\left(k_{1}^{x} v_{j}^{x}\right)^{2}\right. \\
& \times\left(k_{2}^{x} v_{k}^{x}\right)^{2}+\left(k_{1}^{x} v_{j}^{x}\right)^{2}\left(k_{2}^{z} v_{k}^{z}\right)^{2}+\left(k_{1}^{z} v_{j}^{z}\right)^{2}\left(k_{2}^{x} v_{k}^{x}\right)^{2} \\
& \left.\left.+\left(k_{1}^{z} v_{j}^{z}\right)^{2}\left(k_{2}^{z} v_{k}^{z}\right)^{2}+4 k_{1}^{x} v_{j}^{x} k_{1}^{z} v_{j}^{z} k_{2}^{x} v_{k}^{x} k_{2}^{z} v_{k}^{z}\right\}\right\rangle_{0}
\end{aligned}
$$

Проведем усреднение в выражении для $S_{\rho}^{2,2}$, используя (17b), для двухкомпонентной полностью ионизованной водородной плазмы с классической статистикой. Рассмотрим почленно слагаемые из п.п. 1-4. Первое слагаемое из п. 1. имеет вид

$$
\begin{aligned}
& \text { 1.1. }\left(i^{4} / N\right)\left\langle\sum_{i, k, j=1}^{N} e^{3} z_{i} z_{j} z_{k} e^{-i k z_{i}} e^{-i\left(k_{1}^{z} z_{j}+k_{1}^{x} x_{j}\right)} e^{-i\left(k_{2}^{z} z_{k}+k_{2}^{x} x_{k}\right)}\right. \\
& \left.(-) k_{1}^{z} k_{2}^{z} \frac{1}{m_{j} m_{k}} \frac{\partial U}{\partial z_{j}} \frac{\partial U}{\partial z_{k}}\right\rangle_{0}=\beta^{-1} \sum_{i, k, j=1}^{N} e^{3} z_{i} z_{j} z_{k}(-) k_{1}^{z} k_{2}^{z} \\
& \times \frac{1}{m_{j} m_{k}}\left[\frac{\partial U}{\partial z_{j}}\left(-i k_{2}^{z}\right)+\frac{\partial^{2} U}{\partial z_{j} \partial z_{k}}\right] e^{-i\left(k z_{i}+k_{1}^{z} z_{j}+k_{1}^{x} x_{j}+k_{2}^{z} z_{k}+k_{2}^{x} x_{k}\right)} \\
& =\left(i^{4} / N\right) \beta^{-1}\left\langle\sum_{i, k, j=1}^{N} e^{3} z_{i} z_{j} z_{k}(-) k_{1}^{z} k_{2}^{z} \frac{1}{m_{j} m_{k}} \frac{\partial^{2} U}{\partial z_{j} \partial z_{k}}\right. \\
& \left.\times e^{-i\left(k z_{i}+k_{1}^{z} z_{j}+k_{1}^{x} x_{j}+k_{2}^{z} z_{k}+k_{2}^{x} x_{k}\right)}\right\rangle_{0}+\left(i^{4} / N\right) \beta^{-2}\left\langle\sum_{i, k, j=1}^{N} e^{3} z_{i} z_{j} z_{k}\right. \\
& \left.\times\left(k_{1}^{z} k_{2}^{z}\right)^{2} \frac{1}{m_{j} m_{k}} e^{-i\left(k z_{i}+k_{1}^{z} z_{j}+k_{1}^{x} x_{j}+k_{2}^{z} z_{k}+k_{2}^{x} x_{k}\right)}\right\rangle_{0} \quad(18 \mathrm{~b})
\end{aligned}
$$

В термодинамическом пределе определим

$$
\begin{aligned}
U & =\sum_{1 \leq j \leq k \leq N / 2} u_{i j}+\sum_{1 \leq j^{\prime} \leq k^{\prime} \leq N / 2} u_{i^{\prime} j^{\prime}} \\
& +\sum_{1 \leq j^{\prime} \leq k \leq N / 2} u_{j^{\prime} k}+\sum_{1 \leq j \leq k^{\prime} \leq N / 2} u_{j k^{\prime}} .
\end{aligned}
$$

Здесь $(i, j),\left(i^{\prime} j^{\prime}\right)$ соответствуют электронам и ионам, $u$ - кулоновская энергия взаимодействия пар частиц. После усреднения сумм по (17b) (см. подробнее в [6] и ссылки там) получим, отбирая главные члены с $m_{j}, m_{k}=m_{e}$ ( $\hat{k}_{1}^{z}-$ компонента единичного вектора, $N^{\prime}=N / 2$ ), для различных соотношений между индексами $i, j, k: i \neq j \neq k ; j=k ; i=j, i=k ; i=j=k$ (при 
этом свободный индекс может находиться в подсистемах электронов или ионов)

$$
\begin{aligned}
& \left(i^{4} / N\right) \beta^{-1}\left\langle\sum_{i, k, j=1}^{N^{\prime}} e^{3} z_{i} z_{j} z_{k}(-)\left(k_{1}^{z} k_{2}^{z}\right) \frac{1}{m_{j} m_{k}} \frac{\partial^{2} U}{\partial z_{j} \partial z_{k}}\right. \\
& \left.\times e^{-i\left(\mathbf{k r}_{i}+\mathbf{k}_{1} \mathbf{r}_{j}+\mathbf{k}_{2} \mathbf{r}_{k}\right)}\right\rangle_{0}=\frac{e^{3}}{\beta} \frac{k_{1}^{z} k_{2}^{z}}{m_{e}^{2}} \frac{N^{\prime}\left(N^{\prime}-1\right)}{V^{3}} \int\left(\hat{k}_{1}^{z} \cdot \nabla_{1}\right) \\
& \times\left(\hat{k}_{2}^{z} \cdot \nabla_{2}\right) u_{e e}\left(\left|r_{1}-r_{2}\right|\right) e^{-i\left(\mathbf{k r}+\mathbf{k}_{1} \mathbf{r}_{1}+\mathbf{k}_{2} \mathbf{r}_{2}\right)} \\
& \times g_{3 e}\left(r, r_{1}, r_{2}\right) d \mathbf{r} d \mathbf{r}_{1} d \mathbf{r}_{2}+\frac{e^{3}}{\beta} \frac{k_{1}^{z} k_{2}^{z}}{m_{e}^{2}} \frac{N^{\prime}\left(N^{\prime}-1\right)}{V^{3}} \\
& \times \int\left(\hat{k}_{1}^{z} \cdot \nabla_{1}\right)^{2} u_{e e}\left(\left|r_{1}-r_{2}\right|\right) e^{-i\left(\mathbf{k r}+\left(\mathbf{k}_{1}+\mathbf{k}_{2}\right) \mathbf{r}_{1}\right)} \\
& \times g_{3 e}\left(r, r_{1}, r_{2}\right) d \mathbf{r} d \mathbf{r}_{1} d \mathbf{r}_{2}+2 \frac{e^{3}}{\beta} \frac{k_{1}^{z} k_{2}^{z}}{m_{e}^{2}} \frac{N^{\prime}\left(N^{\prime}-1\right)}{V^{3}} \\
& \times \int\left(\hat{k}_{1}^{z} \cdot \nabla_{1}\right)^{2} u_{e i}\left(\left|r_{1}-r_{2}\right|\right) e^{-i\left(\mathbf{k r}+\left(\mathbf{k}_{1}+\mathbf{k}_{2}\right) \mathbf{r}_{1}\right)} \\
& \times g_{e e i}\left(r, r_{1}, r_{2}\right) d \mathbf{r} d \mathbf{r}_{1} d \mathbf{r}_{2}+2 \frac{e^{3}}{\beta} \frac{k_{1}^{z} k_{2}^{z}}{m_{e}^{2}} \frac{N^{\prime}}{V^{2}} \int\left(\hat{k}_{1}^{z} \cdot \nabla_{1}\right) \\
& \times\left(\hat{k}_{2}^{z} \cdot \nabla_{2}\right) u_{e e}\left(\left|r_{1}-r_{2}\right|\right) e^{-i\left(\mathbf{k r}+\left(\mathbf{k}_{1}+\mathbf{k}_{2}\right) \mathbf{r}_{1}\right)} g_{e e}\left(r, r_{1}\right) d \mathbf{r} d \mathbf{r}_{1} \\
& +\frac{e^{3}}{\beta} \frac{k_{1}^{z} k_{2}^{z}}{m_{e}^{2}} \frac{N^{\prime}}{V^{2}} \int\left(\hat{k}_{1}^{z} \cdot \nabla_{1}\right)^{2} u_{e e}\left(\left|r_{1}-r_{2}\right|\right) e^{-i\left(\mathbf{k r}+\left(\mathbf{k}_{1}+\mathbf{k}_{2}\right) \mathbf{r}_{1}\right)} \\
& \times g_{e e}\left(r, r_{1}\right) d \mathbf{r} d \mathbf{r}_{1}+2 \frac{e^{3}}{\beta} \frac{k_{1}^{z} k_{2}^{z}}{m_{e}^{2}} \frac{N^{\prime}}{V^{2}} \int\left(\hat{k}_{1}^{z} \cdot \nabla_{1}\right)^{2} u_{e i} \\
& \times\left(\left|r_{1}-r_{2}\right|\right) e^{-i\left(\mathbf{k r}+\left(\mathbf{k}_{1}+\mathbf{k}_{2}\right) \mathbf{r}_{1}\right)} g_{e i}\left(r, r_{1}\right) d \mathbf{r} d \mathbf{r}_{1} . \\
& \left(i^{4} / N\right) \beta^{-2}\left\langle\sum_{i, k, j=1}^{N} e^{3} z_{i} z_{j} z_{k}\left(k_{1}^{z} k_{2}^{z}\right) \frac{1}{m_{j} m_{k}} e^{-i\left(\mathbf{k r}_{i}+\mathbf{k}_{1} \mathbf{r}_{j}+\mathbf{k}_{2} \mathbf{r}_{k}\right)}\right\rangle_{0} \\
& =(-) \frac{e^{3}}{\beta^{2}} \frac{k_{1}^{z} k_{2}^{z}}{m_{e}^{2}} \frac{N^{\prime}\left(N^{\prime}-1\right)}{V^{3}} \int e^{-i\left(\mathbf{k r}+\mathbf{k}_{1} \mathbf{r}_{1}+\mathbf{k}_{2} \mathbf{r}_{2}\right)} \\
& \times g_{3 e}\left(r, r_{1}, r_{2}\right) d \mathbf{r} d \mathbf{r}_{1} d \mathbf{r}_{2}+(-) \frac{e^{3}}{\beta^{2}} \frac{\left(k_{1}^{z} k_{2}^{z}\right)^{2}}{m_{e}^{2}} \frac{N^{\prime}}{V^{2}} \\
& \times \int e^{-i\left(\mathbf{k r}+\left(\mathbf{k}_{1}+\mathbf{k}_{2}\right) \mathbf{r}_{1}\right)} g_{e e}\left(r, r_{1}\right) d \mathbf{r} d \mathbf{r}_{1}+(-) 2 \frac{e^{3}}{\beta^{2}} \frac{\left(k_{1}^{z} k_{2}^{z}\right)^{2}}{m_{e}^{2}} \frac{N^{\prime}}{V^{2}} \\
& \times \int e^{-i\left(\mathbf{k}+\mathbf{k}_{1}\right) \mathbf{r}+\mathbf{k}_{2} \mathbf{r}_{1}} g_{e e}\left(r, r_{1}\right) d \mathbf{r} d \mathbf{r}_{1}+(-) \frac{e^{3}}{\beta^{2}} \frac{\left(k_{1}^{z} k_{2}^{z}\right)^{2}}{m_{e}^{2}} \\
& \times \frac{N^{\prime}}{V^{2}} \delta_{k+k_{1}+k_{2}} .
\end{aligned}
$$

Второе равенство в (19) соответствует второму выражению в правой части 1.1 (см. (18b)).

Второе, третье и четвертое слагаемые из п. 1 (1.2.-1.4., см. (18a)) после усреднения имеют вид, аналогичный (19). Рассмотрим главный вклад в последнее слагаемое из п. 1. (1.5.), включающее магнитное поле. Этот вклад отличен от нуля при $j=k$.

$$
\begin{aligned}
& \left(i^{4} N\right)\left\langle\sum_{i=1}^{N^{\prime}} e z_{i} e^{-i k z_{i}} \sum_{j=1}^{N^{\prime}} e z_{j} e^{-i\left(k_{1}^{z} z_{j}+k_{1}^{x} x_{j}\right)}\right. \\
& \left.\times \sum_{k=1}^{N^{\prime}} e z_{k} e^{-i\left(k_{2}^{z} z_{k}+k_{2}^{x} x_{k}\right)}\left\{-k_{1}^{x} k_{2}^{x} \frac{e z_{j}}{c m_{j}}\left(v_{j}^{y} H\right)^{x} \frac{e z_{k}}{c m_{k}}\left(v_{k}^{y} H\right)^{x}\right\}\right\rangle_{0} \\
& =\frac{H^{2} N^{\prime}}{V^{2}} k_{1}^{x} k_{2}^{x} \frac{e^{5}}{c^{2}} \frac{1}{\beta m_{e}^{3}} \int e^{-i\left(\mathbf{k r}+\left(\mathbf{k}_{1}+\mathbf{k}_{2}\right) \mathbf{r}_{1}\right)} g_{e e}\left(r, r_{1}\right) d \mathbf{r} d \mathbf{r}_{1} \\
& +\frac{H^{2} N^{\prime}}{V^{2}} k_{1}^{x} k_{2}^{x} \frac{e^{5}}{c^{2}} \frac{1}{\beta m_{e}^{3}} \delta_{k+k_{1}+k_{2} .}
\end{aligned}
$$

В п.п. 2,3 усредненные выражения представляют собой сумму четырех слагаемых, вид которых совпадает с правой частью второго равенства в (19) при значениях произведений векторов перед слагаемыми соответственно $-\left(k_{1}^{x} k_{2}^{x}\right)^{2},-\left(k_{1}^{z} k_{2}^{z}\right)^{2},-\left(k_{1}^{z} k_{2}^{x}\right)^{2},-\left(k_{1}^{x} k_{2}^{z}\right)^{2}$.

В п. 4 усредненное выражение состоит из пяти слагаемых, вид четырех из которых совпадает с правой частью второго равенства в (19) при значениях произведений векторов перед слагаемыми: $\left(k_{1}^{x} k_{2}^{x}\right)^{2},\left(k_{1}^{z} k_{2}^{z}\right)^{2},\left(k_{1}^{z} k_{2}^{x}\right)^{2}$, $\left(k_{1}^{x} k_{2}^{z}\right)^{2}$, пятое слагаемое равно интегралу в (20) с коэффициентом $4 k_{1}^{x} k_{2}^{x} k_{1}^{z} k_{2}^{z} \frac{N^{\prime}}{V^{2}} \frac{e^{3}}{\beta^{2} m_{e}^{2}}$.

При $z_{i}=1$ (соответствующим однозарядным ионам) следует выписать формулы, аналогичные (19) и (20), в которых фигурируют корреляционные функции $g_{e е i}$, $g_{e i i}, g_{e i}$ вместо $g_{3 e}, g_{e e i}, g_{e e}$ и меняется знак перед выражением в правой части. Таким образом, главный вклад в $S_{\rho}^{2,2}\left(\sim 1 / m_{e}^{2}, \sim 1 / m_{e}^{3}\right)$ равен сумме всех главных слагаемых из пп. 1-4, определенных выше. Оценки показывают значительный вклад (20) в коррелятор $S_{\rho}^{2,2}$ при напряженностях постоянного магнитного поля, достижимых в лабораторных экспериментах (параметры плазмы: $n_{e}=10^{17}-10^{21} \mathrm{~cm}^{-3}, T=1-10 \mathrm{eV}, B=10^{4}-10^{5} \mathrm{Gs}$, при которых целесообразно исследовать ПГИ и ГВГ) [5]. Сравним для оценки члены (одинаковые сомножители опущены) в суммах (19) (2-я сумма) и (20) $\left(\frac{e^{3}}{\beta^{2}} \frac{\left(k_{1}^{z} k_{2}^{z}\right)^{2}}{m_{e}^{2}}\right.$ и $\left.\frac{H^{2}}{\beta m_{e}^{3}}\left(k_{1}^{x} k_{2}^{x}\right) \frac{e^{5}}{c^{2}}\right)$; их отношение $\sim \frac{k^{2}}{\beta} / \frac{H^{2} e^{2}}{m_{e} c^{2}}$. Выражая волновой вектор через частоту отсечки и подбирая значения параметров из приведенных выше диапазонов, можно оценить области параметров, где вклад (20) в частотный момент коррелятора $S_{\rho}^{2,2}$ будет незначительным, существенным или преобладающим.

По сформулированной выше методике могут быть рассчитаны другие частотные $S_{\rho}^{r_{1}, r_{2}}$ моменты плотных заряженных сред.

\section{Заключение}

Частотный момент $S_{\rho}^{2,2}$, который наиболее удобно использовать в сформулированной выше модели определения КФР $\hat{\chi}^{(2)}$, проанализирован применительно к 
условиям в лабораторной плотной плазме. Очевидно, что конкретное вычисление главного вклада в частотный момент $S_{\rho}^{2,2}$ по (19), (20) (или других частотных моментов по соответствующим соотношениям, найденным по предложенной в работе методике) для двухкомпонентной полностью ионизованной водородной плазмы с классической статистикой представляет собой отдельную задачу, связанную с заданием межчастичного потенциала взаимодействия (см., например, [8-11,16-19]), корреляционных функций 2-го и 3-го порядков: $g_{e е i}$, $g_{e i i}, g_{e i}$ и $g_{3 e}, g_{e e i}, g_{e e}$ при определенных значениях термодинамических параметров: температуры и давления. Исследованию корреляционных функций из (19), (20) посвящено значительное количество работ (см., например, [8-11,16-19]).

В свою очередь, для явных аппроксимаций квадратичных функций реакции целесообразно использовать выражения, имеющие правильные асимптотики в предельных случаях, например, для разреженной плазмы (см. $[5,14])$. Использование значений одного коррелятора (например, $\left.S_{\rho}^{2,2}\right)$ при некоторых $(P, T, H)$ предполагает однопараметрическую аппроксимацию для КФР. Для определения подгоночных параметров численные значения корреляторов естественно сопоставлять (см. (14)) с моментами свернутых аппроксимаций для тензоров $\hat{\chi}_{p, i j k}^{(2)}$ или $\hat{\chi}_{J, i j k}^{(2)}$.

Заметим, что в работе обсуждался модельный подход к определению квадратичных функций реакции на внешнее поле, основанный, в частности, на вычислении их частотных моментов. При этом использовано соотношение (10), полученное в рамках нелинейной теории отклика на внешнее возмущение $[12,13]$. В то же время при исследовании нелинейных явлений (например, ПГИ и ГВГ) в плазме используют уравнение относительно поляризации среды и электрического поля $\mathbf{E}(\mathbf{r}, t))$ (см., например, [5,14]), в котором фигурирует КФР на среднее поле в данной среде (обозначим их $\chi_{p, i j k}^{(2)}$ и т. п). Поэтому для построения модели для $\chi_{p, i j k}^{(2)}$ по $\hat{\chi}_{p, i j k}^{(2)}$ необходимо уточнение, связанное с подстановкой известного соотношения $\mathbf{D}=\hat{\varepsilon} \mathbf{E}(\hat{\varepsilon}-$ диэлектрическая проницаемость среды) в уравнение, аналогичное (6).

Таким образом, в работе дано обоснование модели для вычисления квадратичных функций реакции, определяющих нелинейные явления, вызванные квадратичным взаимодействием электромагнитных волн в плотной заряженной среде (кулоновские системы, плазма) в постоянном магнитном поле. В данном подходе используется достаточно известная термодинамическая информация о плотных заряженных средах и не требуется существенных компьютерных мощностей по сравнению с реализацией прямого численного моделирования квадратичных функций реакции, в принципе возможного для модельных систем.

\section{Финансирование работы}

Работа выполнена по теме № АAАA-A19119071190017-7.

\section{Конфликт интересов}

Автор заявляет, что у него нет конфликта интересов.

\section{Список литературы}

[1] G. Seibold, M. Udina, C. Castellani, L. Benfatto. Phys. Rev. B, 103, 014512 (2021).

[2] Т.В. Мурзина, И.А. Колмычек, Н.С. Гусев, А.И. Майдыковский. Письма в ЖЭТФ, 111 (6), 370 (2020).

[3] B.M. Goldberg, Tat Loon Chng, A. Dogariu, R.B. Miles. Appl. Phys. Lett., 112, 064102 (2018).

[4] Yu Zhang, Di Huang, Yuwei Shan, Tao Jiang, Zhihong Zhang, Kaihui Liu, Lei Shi, Jinluo Cheng, John E. Sipe, Wei-Tao Liu, Shiwei Wu. Phys. Rev. Lett., 122, 047401 (2019).

[5] Г.А. Павлов. Письма в ЖТФ, 46 (8), 51 (2020). DOI: $10.21883 /$ PJTF.2020.08.49311.18143

[6] Г.А. Павлов. Процессы переноса в плазме с сильным кулоновским взаимодействием (URSS, М., 2019), изд. 2-е, $196 \mathrm{c}$.

[7] Г.А. Павлов, Ю.В. Трощиев. Нелинейная теория отклика и гидродинамика плотных сред с источниками (URSS, М., 2019), 152 c.

[8] Р.Л. Стратонович. Нелинейная неравновесная термодинамика (Наука, М., 1985).

[9] Book of Abstracts of SCCS 2017, July 30-4 August 2017, Kiel, Germany (http://www.uni-kiel.de/sccs2017/).

[10] T. Dornheim, J. Vorberger, M. Bonitz. Phys. Rev. Lett., 125, 085001 (2020).

[11] T. Dornheim, Z. Moldabekov, J. Vorberger. J. Chem. Phys., 155, 054110 (2021).

[12] Р. Кубо. Некоторые вопросы статистическомеханической теории необратимых прочессов. В сб. Термодинамика необратимых процессов, пер. с англ. (Наука, М., 1962)

[13] Д.Н. Зубарев. Неравновесная статистическая термодинамика (Наука, М., 1971)

[14] А.Г. Ситенко. Флуктуации и нелинейное взаимодействие волн в плазме (Наукова Думка, Киев, 1977)

[15] Л.Б. Мейснер, С.М. Салтиел. Нелинейные восприимчивости: экспериментальные данные и методы расчета. Справочник по лазерам в 2 m. под ред. А.М. Прохорова. (Сов. Радио, М., 1978), т. 2, с. 271-292.

[16] И.З. Фишер. Статистическая теория жиикостей (Физматлит, М., 1961)

[17] C. Deutsch. Phys. Lett. A, 60, 317 (1977).

[18] Теплофизические свойства рабочих сред газоббазного ядерного реактора, под ред. В.М. Иевлева (Атомиздат, M., 1980)

[19] Физика простых жсидкостей, под ред. Г. Темперли, Дж. Роулинсона, Дж. Рашбрука (Мир, М., 1971) 